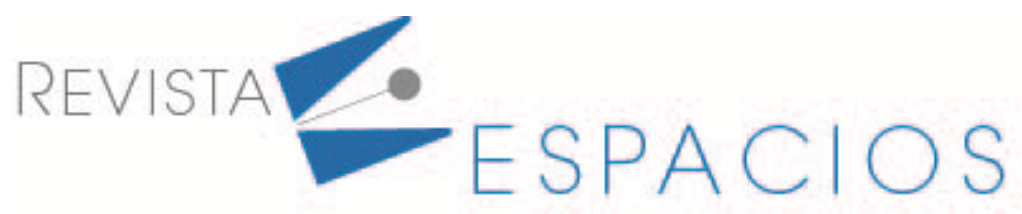

\title{
Trayectoria investigativa en docentes de ciencias naturales: dos experiencias
}

\author{
Research trajectory in natural science teachers: two experiences
}

\author{
SANABRIA TOTAITIVE, Inés Andrea ${ }^{1}$ \\ MORENO CELY, Claudia Patricia ${ }^{2}$ \\ ARANGO MARTíNEZ, Angie Viviana ${ }^{3}$
}

\begin{abstract}
Resumen
El propósito del estudio fue indagar la trayectoria investigativa de dos docentes de Ciencias Naturales que se desempeñan en el departamento de Boyacá-Colombia, a partir de una metodología cualitativa utilizando la investigación biográfico-narrativa. El análisis se hizo a través de la reconstrucción de experiencias de formación inicial y permanente, la práctica pedagógica y el contexto donde laboran, esto demostró que los docentes que implementan la investigación en el aula reflexionan sobre su acción para construir conocimiento y perfeccionar su rol.

Palabras clave: investigación, docente, formación
\end{abstract}

\begin{abstract}
The purpose of this study was to investigate the research trajectory of two teachers of Natural Sciences who work in the department of Boyacá-Colombia, since a qualitative methodology using biographical-narrative research. The analysis was done through the reconstruction of initial and ongoing training experiences, pedagogical practice and the context where they work, this showed that teachers who implement research in the classroom, reflect on their action in order to build knowledge and improve their role.

key words: research, teacher, training
\end{abstract}

\section{Introducción}

La investigación científica en la realidad escolar debe ser vista como procesos de formación y medio para desarrollar estilos de pensamiento con el fin de mejorar la calidad de vida de las personas y comunidades, puesto que en los distintos contextos escolares surgen necesidades que conllevan a cambios, los cuales deben responder a los avances de la ciencia, la tecnología, la sociedad, la educación y la cultura, de tal manera, que genera en los docentes la posibilidad de despertar, fortalecer y mantener en los niños y jóvenes la capacidad de curiosidad y de asombro, al formar en ellos actitudes abiertas y flexibles ante las realidades que vive el país.

Al incursionar en el mundo de la profesión docente, los maestros suelen preguntarse ¿para qué investigar en el aula?, respuesta que varios autores (Tomé, 2015; Manzano, 2015; Araque, 2019), han atribuido al contexto de

\footnotetext{
${ }^{1}$ Estudiante del Doctorado en Educación Sociedad. Magister en Educación. Docente de la Universidad Pedagógica y Tecnológica de Colombia. Grupo de Investigación de Estudios en Ecología, Etología, Educación y Conservación. inesandreasanabria@uptc.edu.co

${ }^{2}$ Magister en Educación. Docente de la Universidad Pedagógica y Tecnológica de Colombia. Grupo de Investigación de Estudios en Ecología, Etología, Educación y Conservación. claudia.moreno@uptc.edu.co

${ }^{3}$ Licenciada en Ciencias Naturales y Educación Ambiental. Universidad Pedagógica y Tecnológica de Colombia. Grupo de Investigación de Estudios en Ecología, Etología, Educación y Conservación. angie.arango@uptc.edu.co
} 
la época contemporánea, la cual se caracteriza por las innovaciones, renovaciones y reformas, pero también trae incertidumbres que requieren de cambios significativos para mejorar la calidad formativa de la práctica pedagógica; entonces, la investigación como base de la educación, se convierte en la posibilidad para estudiar la diversidad de estudiantes, educadores, directivos, comunidades y estrategias metodológicas tanto de enseñanza como de aprendizaje.

En este sentido, los docentes de las instituciones educativas a parte de apropiarse del saber pedagógico y disciplinar, deben manejar el saber científico desde la escuela o también llamado saber investigativo (Bolaños, 2016), es decir, el docente de hoy en día debe ser un investigador de su sociedad y así buscar una reflexión de su propia práctica (Fernándes, 2014; Bolaños, 2016). Está característica de ser un docente investigador se suma a las multiples funciones de su labor (Duarte, 2007), donde enseñar contenidos ya no es suficiente para que los estudiantes asuman los cambios del contexto, es así, que se hace necesario incluir en la educación de calidad, según Alarcón, Montes y Múnera (2019) acciones que lleven al desarrollo de competencias en los estudiantes y al desenvolvimiento social.

La investigación en el aula es también llamada investigación educativa, y aunque incluirla en la práctica docente conlleva dificultades para innovar como la desmotivación por parte de los docentes, la deficiente formación en cuanto a didácticas pedagógicas, la falta de trabajo cooperativo y de tiempo (Peláez y Piedrahita, 2018), también son muchos los beneficios que la investigación trae a la escuela, entre ellos se pueden mencionar, que la experiencia de investigar en el aula se convierte en una estrategia pedagógica que permite enseñar, autoformar, integrar áreas, dialogar y trabajar cooperativamente no solo en grupos de estudiantes sino también de docentes y comunidades, dándoles la oportunidad de construir conocimiento a partir de sus prácticas pedagógicas (Plata, 2015); además, la investigación educativa permite y obliga a los docentes a mantenerse actualizados sobre su contexto y los contenidos, llevándolos a un proceso de formación permanente profesional para mejorar la enseñanza y generar conocimiento (Muñoz y Garay, 2015).

Sin embargo, muchos de los procesos investigativos realizados por los docentes, no son sistematizados ni compartidos con la comunidad científica, afectando así su trascendencia y significancia investigativa, es por ello, y según Porlán (2011) que se requiere de tres componentes indispensables y simultáneos para investigar situaciones del aula como el contexto social, la conducta del niño y del profesor, el aprendizaje de conceptos y de actitudes, la validez de recursos didácticos, entre otras; estos componentes son: el "querer", el "saber" y el "poder". Así como Porlán, otros autores han coincidido en que la motivación y voluntad del profesor son el motor que genera la acción (Porlán, 2011; Peláez y Piedrahita, 2018), pero el "querer" no es suficiente, el docente debe saber fomentar la indagación y el descubrimiento, saber de qué tipo es la investigación y qué sentido tiene, también cómo se hace y que ésta debe ser compartida con los compañeros, por último, "querer" y "saber" tampoco son suficientes, el docente debe "poder" hacer investigación, y para ello, requiere de tiempo, comunidades críticas, condiciones organizativas determinadas, ayudas y medios necesarios, así como de autonomía para investigar (Porlán, 2011).

Entonces para investigar en la escuela, influye la formación del docente tanto inicial como permanente, y en esa formación inicial se ve involucrada la universidad y los ámbitos de formación investigativa que esta proporcione, uno de estos ámbitos son los grupos de investigación, los cuales son definidos, en Colombia, como una estrategia extracurricular para la formación de investigadores desde el pregrado, se caracterizan por ser espacios agradables y de participación voluntaria, donde se generan preguntas orientadas al desarrollo de competencias investigativas y un enriquecimiento académico y personal, es así, que los grupos de investigación se han convertido en espacios en los que el estudiante pasa del modelo tradicional académico a uno de aprendizaje que nace de la discusión, la iniciativa propia y el aprender a investigar (Saavedra, Muños, Antolínez, Rubiano y Puerto, 2015). 
Para el 2019, Colombia reporta 5.772 grupos de investigación reconocidos ante Minciencias en sus diferentes categorías (A1, A, B, C y Reconocido), dónde el $40 \%$ de los grupos se encuentran en categoría C, de estos datos se tiene que el 38\% (2.170) de los grupos de investigación son del Programa Nacional Ciencia, Tecnología e Innovación en Ciencias Humanas, Sociales y Educación; según el Gran Área de Conocimiento el 18\% (1.034) de los grupos son de Ciencias Naturales y teniendo en cuenta el Área de conocimiento de Ciencias de la Educación, de los 415 grupos de investigación, 173 se encuentran categorizados en C (Minciencia, 2020). Esto da cuenta de la gran influencia que la formación investigativa tiene desde los pregrados universitarios, brindando la posibilidad de que docentes y docentes en formación se conviertan en investigadores de su propia práctica para reflexionar y mejorarla, construyendo no solo conocimiento, sino también metodológicas, experiencias y habilidades tanto académicas como personales.

Ya en la práctica docente, la investigación de las escuelas en Colombia, es incentivada desde la participación del programa Ondas Minciencias, que desde su creación en el año 2001, ha promovido la participación de más de 4 millones de estudiantes (niños, niñas y jóvenes), organizados en más de 8.000 grupos de investigación de Instituciones Educativas y a los cuales se han vinculado más de 100 mil docentes; es así, como la investigación se convierte en una estrategia pedagógica y metodológica que lleva a la construcción de habilidades sociales, cognitivas y comunicativas, también de capacidades para ordenar la indagación; además, los temas de estudio surgen de pequeñas preguntas generadas en el aula desde cada contexto y con la orientación del docente se van convirtiendo en verdaderos problemas para investigar, donde la experiencia es un factor importante para la construcción de saber mediado por la reflexión de la misma, dándole sentido y significado a las prácticas docentes (Plata, 2015; Minciencia, 2020).

De acuerdo a lo anterior, estudios como los de González, Zerpa, Gutiérrez y Prieto (2007) se han preocupado por caracterizar la importancia que tiene la investigación educativa en el hacer docente, en el sentido en que, cuando el docente investiga en y sobre su acción se convierte en un investigador en el aula, en la escuela y en su comunidad, donde la propia reflexión es el mejor instrumento para un aprendizaje significativo y para un apropiado desarrollo profesional. Sin embargo, otros autores destacan que según la investigación sobre la formación del profesorado y la didáctica de las ciencias, son pocos los docentes que muestran interés por leer e intentan adaptar los resultados de las investigaciones educativas con la intención de mejorar su práctica (Parga, 2016; Vázquez y Manassero, 2019).

Una de las formas para realizar y mostrar este tipo de investigaciones, ha sido adoptar la metodológia de investigación biográfico-narrativa. En los últimos años el desarrollo de la investigación narrativa ha ido creciendo y sobre todo en el contexto educativo, ya que es una manera de exponer las propias voces y así tener una aproximación con las acciones; además las narrativas permiten representar realidades vividas, pues a partir del diálogo y la indagación, la realidad se convierte en texto, construyendo así entre participantes y el investigador los datos que serán analizados en el proceso (Arias y Alvarado, 2015). La investigación biográfico-narrativa involucra herramientas y técnicas dentro de su metodología como lo son: la indagación sobre las historias de vida, observaciones participantes y no participantes, grupos de discusión, entre otras, las cuales son una posibilidad para acceder a conocimientos y experiencias de los participantes y que permitirán realizar una construcción basada en la reflexión de los mismos (Bolívar, Domingo y Fernández, 2001; Yedaide, Álvarez y Porta, 2015)

En este sentido, la investigación que aborda el presente escrito, tuvo como propósito indagar sobre la trayectoria investigativa de dos docentes en ejercicio de ciencias naturales, y así realizar una construcción de sus experiencias, de acuerdo a su formación tanto inicial como permanente, a su práctica pedagógica y al contexto desde el cual actúan. 


\section{Metodología}

El estudio que se aborda desde la metodología cualitativa (Hernández, Fernández y Baptista, 2006), y la hermenéutica, buscó interpretar y comprender la formación de la investigación en el contexto de sus prácticas pedagógicas a través de la investigación biográfico-narrativa, que de acuerdo con Bolívar et al., (2001), es una metodología autobiográfica con un enfoque narrativo, y estos a su vez son un enfoque de investigación y una práctica de formación, en donde se facilita la descripción de experiencias, de saberes y de competencias profesionales.

En este sentido, la narración remite a la vida, leer es un modo de vivir, contar y leer narraciones es vivirlas en el mundo de lo imaginario, recreándolas en uno, puesto que el proceso de composición se realiza en el lector, a partir de la fusión de horizontes: entre el horizonte de su propio mundo del lector y el mundo de la ficción; la vida misma remite a la narración, la vida no es solo biología, sino la concreción en un bios - una biografía o un relato (Mélich y Bárcena, 2014). Partiendo que una historia de vida no es solo una recolección de recuerdos pasados, ni una ficción, es una reconstrucción desde el presente (identidad del yo) en función de una trayectoria futura, interpretar el texto de la acción, es para el agente, interpretarse así mismo (Ricoeur, 1996). Según Ricoeur, narrar la historia de nuestra vida es una autointerpretación de lo que somos, una puesta en escena a través de la narración (Bolívar et al., 2001), de esta manera, la hermenéutica de la vida humana.

A su vez, Ricoeur especifica que el educador no sabe lo que hace hasta que las acciones hacia una actividad pedagógica finalizan y puede construir un relato o narración, a través del recuerdo reflexivo, el saber de la acción solo se puede alcanzar cuando, al dejar de actuar, pierde el agente su condición de tal y se convierte en el personaje del relato que otro cuenta. La identidad personal no puede articularse más que en la dimensión temporal de la vida, que cobra existencia en el relato narrativo (Ricoeur, 1996).

En este sentido, el estudio realizado involucró la trayectoria investigativa de dos docentes de ciencias naturales a quienes se les denomina Juan y Pedro (pseudónimos), los cuales pertenecen al Magisterio en Instituciones Educativas de diferentes municipios del departamento de Boyacá-Colombia. Para indagar sobre sus experiencias, se acudió a la entrevista semiestructurada con enfásis narrativo, las cuales fueron grabadas y transcritas para su posterior análisis de codificación y categorización. El tipo de análisis de los datos, tuvo un enfoque categórico del contenido, el cual es familiar para el análisis de contenido, a su vez para el análisis de la entrevista se asumió una postura analítica y de reconstrucción del sentido, la cual incluyó el análisis profundo de las entrevistas para determinar categorías y dar un sentido a la práctica de investigación en la labor docente (Bolívar et al., 2001). Las categorías de análisis se describen a continuación:

- Contexto: definido como escenario físico, geográfico, temporal, histórico, cultural y estético, en donde la acción se desarrolla, es utilizado para ubicar a las personas y los eventos en un tiempo y espacio determinados, de tal manera, que se convierte en un recurso para comprender lo que sucede y lo que las personas dicen y hacen (Giraldo, 2013), en este sentido, en el campo educativo es considerado como una posibilidad para que el docente articule la institución, el currículo y los entornos del estudiante con el proceso de enseñanza aprendizaje (Aaron, 2016).

- Formación inicial y permanente: la formación da lugar a estructurar un sujeto desde todas sus dimensiones a partir de lo social y lo cultural, respondiendo al ser desde su vocación, al saber desde lo profesional y al saber hacer desde su ocupación lo que conlleva a una coherencia de vida. En razón a lo anterior, y según el MEN (2013) "el sujeto en formación se enfrenta a distintos ámbitos del conocimiento disciplinar, pedagógico, ético, estético, investigativo, comunicativo, personal, social y cultural; requeridos para asumir la labor de un educador profesional en los niveles nacional, regional y local" 
(p.60). Es importante resaltar que el presente artículo, se centrará en analizar el ámbito investigativo de la formación inicial y permanente de los participantes.

- Práctica pedagógica: esta orienta la reflexión que realiza el docente sobre su acción, es decir, sobre su práctica pedagógica (Páez, 2015), es así, que esta categoría expone los procesos de mediación, que incluye la investigación, realizados por el docente para mejorar la enseñanza.

- Experiencia: la experiencia pedagógica, puede definirse de diferentes maneras según su naturaleza de: enseñanza, experimento, habilidad y sentimiento; en este artículo se entiende como los intentos que realiza el docente en su enseñanza, que le permite hacer ajustes, corregir y mejorar su quehacer (experiencia pedagogía-conocimiento), y como una experiencia que se tiene sobre el conocimiento de las cosas y del mundo, producto de una enseñanza asociada a la pedagogía, la didáctica, la institución educativa, el enseñante, y también con los aprendizajes de los padres de familia en los distintos ciclos vitales del ser humano, en particular la infancia, la niñez y la adolescencia (Páez, 2015), donde se ve reflejada en el actuar del docente y de sus estudiantes, es decir, en las actividades investigativas que realizan involucrando el contexto, la familia y el conocimiento disciplinar de la escuela.

\section{Resultados}

En los resultados se muestra la reconstrucción de los acontecimientos más significativos de la vida profesional e investigativa de los profesores Juan (cuadro 1) y Marcos (cuadro 2), estos fueron organizados en forma de bitácoras, según lo expuesto por Bolívar et al., (2001), cada una ordenada en tres columnas las cuales se leen de izquierda a derecha: en la columna del centro se evidencia a manera de resumen los acontecimientos relevantes del proyecto de vida de los participante, en la primera columna se presenta una aproximación de la fecha en la que ocurrieron los hechos, y la tercera columna refleja la valoración que las autoras realizan acerca de los sucesos.

\section{Cuadro 1}

Bitácora sobre la trayectoria investigativa del docente Juan.

\begin{tabular}{|c|c|c|}
\hline \multicolumn{3}{|c|}{ JUAN } \\
\hline Fecha & Acontecimiento & Valoración \\
\hline $\begin{array}{c}\text { Pregrado } \\
2000-2006\end{array}$ & $\begin{array}{l}\text {-Integrante de un grupo de investigación desde los inicios del } \\
\text { pregrado. } \\
\text {-Participación en diferentes eventos científicos a nivel nacional. } \\
\text {-Participación en el proceso de reconocimiento del grupo ante } \\
\text { Minciencias. } \\
\text {-Participación en la formulación de proyectos y escritura científica. } \\
\text {-Licenciado en Ciencias Naturales y Educación Ambiental. } \\
\text {-Tuvo como mentora a una docente de la universidad que lo } \\
\text { orientó y lo motivó a incluir la investigación en su vida. }\end{array}$ & $\begin{array}{l}\text {-La investigación en el } \\
\text { pregrado promovió la } \\
\text { formación profesional y } \\
\text { personal del docente. } \\
\text {-Universidad Pública. }\end{array}$ \\
\hline 2007 & $\begin{array}{l}\text {-Joven investigador Minciencias. } \\
\text {-Promueve procesos de formación de semilleros de investigación. } \\
\text {-Capacitación a docentes de Instituciones Educativas. } \\
\text {-Acompañamiento al programa Ondas Minciencias. } \\
\text {-Becario de la Red Latinoamericana de Etnobotánica. } \\
\text {-Curso de formación en Honduras sobre plantas. } \\
\text {-Publicación de artículos. }\end{array}$ & $\begin{array}{l}\text {-Acciones académico - } \\
\text { investigativas que } \\
\text { promovieron la formación } \\
\text { permanente del docente. }\end{array}$ \\
\hline
\end{tabular}




\begin{tabular}{|c|l|l|}
\hline \multicolumn{2}{|c|}{ JUAN } \\
\hline Fecha & \multicolumn{1}{|c|}{ Acontecimiento } & \multicolumn{1}{|c|}{ Valoración } \\
\hline \multirow{2}{*}{$\begin{array}{c}\text { Posgrado } \\
2008-2009\end{array}$} & $\begin{array}{l}\text {-Maestría en Administración de la Informática Educativa. } \\
\text {-Trabaja como docente en una Institución Educativa pública rural } \\
\text { de telesecundarias. } \\
\text {-Realiza procesos de investigación con sus estudiantes vinculando } \\
\text { el contexto rural. }\end{array}$ & $\begin{array}{l}\text {-Compartió conocimientos } \\
\text { del posgrado con sus } \\
\text { estudiantes en el desarrollo } \\
\text { de las clases con el fin } \\
\text { promover la investigación } \\
\text { en el aula. }\end{array}$ \\
\hline \multirow{2}{*}{ 2010-actual } & $\begin{array}{l}\text {-Participación de los estudiantes en convocatorias y becas con los } \\
\text { proyectos realizados en la escuela. } \\
\text {-Rector de la Institución Educativa donde siempre ha laborado. }\end{array}$ & $\begin{array}{l}\text {-Promueve en sus } \\
\text { estudiantes la investigación } \\
\text { y el gusto por aprender. }\end{array}$ \\
\hline
\end{tabular}

Fuente: elaboración propia basada en Bolívar et al (2001).

Cuadro 2

Bitácora sobre la trayectoria investigativa del docente Marcos.

\begin{tabular}{|c|c|c|}
\hline \multicolumn{3}{|c|}{ MARCOS } \\
\hline Fecha & Acontecimiento & Valoración \\
\hline $\begin{array}{c}\text { Pregrado } \\
2002-2006\end{array}$ & $\begin{array}{l}\text {-Perteneciente al grupo de investigación desde que inició el pregrado, } \\
\text { realizó actividades de organización, participación, y formación como } \\
\text { semillero. } \\
\text {-Participación en el Congreso de Botánica en Cartagena. } \\
\text {-Participó de la Asociación Colombiana de Ciencias Biológicas en Ibagué. } \\
\text {-Participó en el congreso Internacional de Formación de Docentes } \\
\text { realizado en la Universidad de Antioquia. } \\
\text {-Pasante de Acerías Paz del Río en la evaluación de las plantaciones de } \\
\text { eucalipto. } \\
\text {-Tuvo como mentora a una docente de la universidad que lo orientó y lo } \\
\text { motivó a incluir la investigación en su vida. }\end{array}$ & $\begin{array}{l}\text { La investigación en el } \\
\text { pregrado promovió la } \\
\text { formación profesional } \\
\text { y personal del docente. } \\
\text {-Universidad Pública. } \\
\text {-Acciones académico - } \\
\text { investigativas que } \\
\text { promovieron la } \\
\text { formación permanente } \\
\text { del docente. }\end{array}$ \\
\hline $\begin{array}{c}\text { Actividades } \\
\text { de docencia } \\
\text { e } \\
\text { investigación } \\
2007 \text { - } 2015\end{array}$ & $\begin{array}{l}\text {-Ingresa al Magisterio en Puerto Boyacá en el Colegio Puerto Pinzón, } \\
\text { cerca de la reserva de aves del Paujil de pico azul. } \\
\text { - Al iniciar su proceso de docente, se encuentra con obstáculos para } \\
\text { incluir la investigación en la institución educativa. } \\
\text {-Realiza procesos de innovación e investigación en el aula, como: crear el } \\
\text { Club Escolar de Investigación y Producción Audiovisual. } \\
\text {-Implementa la fotografía como estrategia pedagógica para la } \\
\text { autoevaluación de su practica docente y para promover la lectura y la } \\
\text { escritura en los estudiantes. } \\
\text {-Pasa al Guicán de la Sierra, al colegio Normal Superior de Nuestra } \\
\text { Señora del Rosario. } \\
\text {-Allí crea los Héroes del Planeta en la Conservación del Cóndor Andino y } \\
\text { Los Héroes del Planeta Van a la Escuela como estrategias para dinamizar } \\
\text { el aprendizaje de las ciencias y compartir los proyectos realizados con los } \\
\text { estudiantes. } \\
\text {-En Guicán comienza con el programa de Ondas Ambientales y Moléculas } \\
\text { de Vida como estrategia para dar a conocer a la comunidad las } \\
\text { actividades que se realizaban en el grupo semillero de investigación. } \\
\text {-Fue experiencia a mejor práctica en innovación a nivel de Boyacá, esto le } \\
\text { permitió ir a Cuba a participar en el Congreso de Pedagogía del año } 2011 \\
\text { representando a Colombia. } \\
\text {-De a cuerdo al trabajo realizado en Guicán, fue experiencia de Boyacá en } \\
\text { fomentar la lectura y escritura utilizando herramientas audiovisuales. }\end{array}$ & $\begin{array}{c}\text {-Compartió y } \\
\text { enriqueció los saberes } \\
\text { con sus estudiantes a } \\
\text { través de la vivencia de } \\
\text { experiencias } \\
\text { aprendidas en su } \\
\text { constante formación } \\
\text { académico- } \\
\text { investigativo } \\
\text {-Promueve en sus } \\
\text { estudiantes la } \\
\text { investigación y el gusto } \\
\text { por aprender. } \\
\text {-Valoró y contribuyó a } \\
\text { los procesos } \\
\text { pedagógicos didácticos } \\
\text { en los distintos } \\
\text { entornos escolares en } \\
\text { los que ejerció y ejerce, } \\
\text { a partir del }\end{array}$ \\
\hline
\end{tabular}




\begin{tabular}{|c|c|c|}
\hline \multicolumn{3}{|c|}{ MARCOS } \\
\hline Fecha & Acontecimiento & Valoración \\
\hline & $\begin{array}{l}\text {-Desarrolla festivales con los estudiantes en los que hace reconocimiento } \\
\text { del territorio. } \\
\text {-Crea la estrategia de comunicación comunitaria de producción } \\
\text { audiovisual Eco Parches (El Parche), la cual es manejada en la mayoría de } \\
\text { los Parques Nacionales del país. } \\
\text {-En el año } 2013 \text { pasa a laborar en una postprimaria de Siachoque llamada } \\
\text { San José, luego pasa a ser parte del colegio del área urbana Ignacio Gil } \\
\text { Sanabria del municipio de Siachoque. } \\
\text {-En el año } 2015 \text { fue mejor experiencia Nacional en Innovación y Educar } \\
\text { con Tic, lo que le permitió viajar a Corea. } \\
\text {-Realiza crónicas y audio cuentos con los estudiantes, proceso que es } \\
\text { acompañado por el concurso nacional del cuento de RCN y el Ministerio. }\end{array}$ & $\begin{array}{l}\text { conocimiento } \\
\text { adquirido en sus } \\
\text { prácticas pedagógicas, } \\
\text { reflejadas en las } \\
\text { estrategias } \\
\text { metodológicas de } \\
\text { enseñanza que ha } \\
\text { creado basadas en la } \\
\text { investigación en el } \\
\text { aula. }\end{array}$ \\
\hline $\begin{array}{c}\text { Actividades } \\
\text { de docencia } \\
\text { e } \\
\text { investigación } \\
\text { 2016-Actual }\end{array}$ & $\begin{array}{l}\text {-Crea una secuencia didáctica en Siachoque, en la que se lee, escribe, se } \\
\text { documenta, luego se hace el guión, graba el audio, se hacen las tomas y } \\
\text { edición de los videos y por último se comparte en redes, además permite } \\
\text { integrar diferentes áreas. } \\
\text {-El trabajo de investigación que venía realizando lo configura en un } \\
\text { proyecto que titula Tras las Huellas del Agua, Conservamos Nuestro } \\
\text { Territorio y tiene tres líneas: Investigación, Educación Ambiental y } \\
\text { Comunicación. } \\
\text {-El proceso investigativo se ha sistematizado en el libro “CUIDANDO AL } \\
\text { PLANETA DESDE LA ESCUELA”. } \\
\text {-En el año 2016, la Secretaría de Educación, gobernación de Boyacá } \\
\text { replica todo el trabajo de producción audiovisual y crea el Festival Escolar } \\
\text { Audiovisual, Fescol Boyacá. } \\
\text {-Tras las Huellas del Agua, Conservamos Nuestro Territorio fue } \\
\text { experiencia nacional en pragmática de la Educación Ambiental en el año } \\
2018 . \\
\text {-Desde el año } 2019, \text { está en Nuevo Colón, donde promueve la } \\
\text { investigación desde el Club Corpochivor el Parche. } \\
\text {-Brinda conferencias y charlas sobre su proceso de investigación con los } \\
\text { estudiantes. } \\
\text {-El Club de Corpochivor el Parche, fue seleccionado como el mejor Club, } \\
\text { por todo el trabajo de comunicación que realizan. } \\
\text {-Los Héroes del Planeta Van a la Escuela, fue seleccionado por Boyacá } \\
\text { Minciencias en pro de la celebración de los } 15 \text { años del programa Ondas. } \\
\text {-En el } 2019 \text { son reconocidos como experiencia Departamental en } \\
\text { Currículo Ambiental. } \\
\text {-Hoy en día algunos de sus estudiantes que se formaron en investigación, } \\
\text { tienen profesiones relacionadas con la educación ambiental. }\end{array}$ & \\
\hline
\end{tabular}

Fuente: elaboración propia basada en Bolívar et al (2001).

Por otra parte, se presentan cada una de las categorías seleccionadas a analizar, estas son: contexto (Contexto y posibilidades en la enseñanza aprendizaje), formación investigativa (inicial y permanente), práctica pedagógica (La práctica pedagógica como escenario de investigación) y experiencia (La Experiencia refleja los procesos investigativos).

\subsection{Contexto y posibilidades en la enseñanza aprendizaje}

El contexto en el cual los docentes se forman investigativamente, cobra gran importancia cuando inician su labor, donde los retos, dificultades pero también posibilidades con las que se encuentran son aprovechados y 
solventados con procesos de investigación, permitiéndoles estar en una formación y reflexión continua sobre su práctica. Tanto el docente Juan como el docente Marcos se formaron en una universidad pública y desde sus inicios del pregrado se involucraron en procesos investigativos, han trabajado en diferentes ámbitos rurales algunos con modalidad multigrado, como lo menciona el docente Juan:

“...trabajo como profesor en una Institución Educativa de carácter público de teles-secundaria, donde soy el único docente de los grados sexto hasta noveno, con el tiempo se fueron modificando ahorita lo llamamos secundaria activa y media rural, esas son aulas multigrado las cuales tenemos todos los grados y tenemos todas las áreas a nuestro cuidado, los Licenciados en Ciencias Naturales...", así mismo, el docente Marcos: “...estando en Siachoque pasó a laborar una pos primaria y como pos primaria junto con otras dos compañeras allí me tocó llegar a orientar de sexto a noveno las diferentes áreas inglés, informática, matemáticas, naturales...".

En este sentido, el contexto actual en el cual se sitúa la educación, es un contexto que exige diversos aspectos que se deben tener en cuenta, entre ellos, el saber vincularlo a los procesos de enseñanza aprendizaje. El docente Marcos es un ejemplo de ello, puesto que ha trabajado en diferentes Instituciones educativas las cuales proporcionan un ambiente rural con áreas protegidas para vincular la investigación y orientar la comprensión del saber disciplinar, resaltando la importancia de incluir la escuela con el entorno y la comunidad, al respecto menciona: "...entonces yo tengo acá: Reserva de la Sociedad Civil, Parque Nacional Natural, Parque Natural Regional y ahora Distrito de Riesgo de Manejo Integrado; como las cuatro figuras ambientales que existen de áreas protegidas, y entonces estamos ahí vinculando a la escuela y todo..." (Marcos).

Se evidencia la importancia que los docentes aluden a conocer y aprender del contexto, viéndolo como una forma de acercar los estudiantes al aprendizaje para comprender su realidad, así mismo, Aarón (2016) menciona que analizar el contexto y sus diversos aspectos, permite tener una mirada clara sobre los estudiantes y su entorno más cercano para vincularlo con los procesos de enseñanza aprendizaje.

\subsection{Formación inicial y permanente del docente investigador}

La formación del docente involucra los saberes recibidos en la universidad y el desempeño de la profesión docente, en la mayoría de los casos la formación docente (personal, teórica, disciplinar, investigativa y en valores) concluye en la escolaridad al no contar con un plan permanente, dando origen a la rutina, al conformismo y al abandono intelectual (Díaz, 2006). Los docentes participantes del presente estudio, continúan en su proceso formativo articulado a la investigación, además, se le suma la participación en eventos científicos regional, nacional e internacional como se muestra en los cuadros 1 y 2 , abriendo oportunidades para adquirir becas de estudio, por ejemplo: “...fui experiencia a nivel de Boyacá como la mejor práctica en innovación lo que me permitió ir a Cuba representando al país..." (Marcos).

De igual manera, se evidencia la percepción sobre investigación que el docente Juan ha creado de acuerdo a su experiencia: "...la investigación realmente es lo que nos permite desarrollar otros campos de nuestra formación como docentes no es solo como decir una forma de motivar, sino es una forma más fácil de enseñar, incluso de compartir saberes..."; el docente resalta como la investigación facilita y mejora los procesos de enseñanza aprendizaje (Muñoz y Garay, 2015) a partir de la reflexión de su práctica le es posible complementar los diferentes ámbitos de su formación, destacando un aprendizaje continuo, cooperativo y comunitario, "...yo publico un articulo "La investigación, la forma más sencilla de compartir saberes y experiencias", a partir de eso no solo se ve que el docente es el que va y sabe y él que da sus clases y que ya, no, con ese proceso también compartimos lo que saben los estudiantes, lo que saben los papás, lo que sabe la comunidad, entonces es un proceso continuo de conformación diría yo, no solo es que el docente es el único que sabe..." (Juan), en este 
sentido, Muñoz y Garay (2015) afirman que la investigación es un proceso permanente que aporta al desarrollo profesional y empoderamiento del rol docente.

La investigación además, es un proceso voluntario, el docente Marcos menciona que la investigación la realizan

“...algunos, los que quieran, porque esto lo hacemos también por fuera del colegio, igual que cuando uno está en Gecos (grupo de Estudios enEcología, Etología, Educacióny Conservación) uno sale y hace por fuera de sus clases y de sus cosas, esto es voluntariado...", de acuerdo con Porlán (2011) se hace referencia a la voluntad como motor decisivo para generar la acción investigativa, es decir, el querer hacer, pero, para darle sentido a la investigación en la escuela es necesario querer, saber y poder simultáneamente.

En este sentido, el proceso de formación investigativa de los docentes Juan y Marcos, ha permitido enriquecer sus conocimientos teóricos aplicándolos a la práctica, además, han adquirido destrezas y habilidades para construir conocimiento, cambiar discursos, metodología y métodos utilizados en el aula, transformando de esta manera los procesos de enseñanza (Muñoz y Garay, 2015), lo que significa que la formación se constituye en un sinónimo de desarrollo y proyección profesional del educador, siendo posible desde su desempeño hacer una mirada crítica e integral del sistema educativo (MEN, 2013, p. 49), de igual forma, la investigación y la formación docente son determinantes para buenas prácticas pedagógicas (Alarcón et al., 2019).

\subsection{La práctica pedagógica como escenario de investigación}

Los docentes participantes demuestran incluir la investigación en sus procesos de enseñanza en el aula, se evidencia que ponen en práctica la formación investigativa recibida desde el pregrado, hasta los cursos y posgrados que han venido desarrollando a lo largo de su vida profesional, al respecto el docente Marcos menciona: "...la metodología de grupo, la sigo aplicando pues no es un semillero de investigación pero es un Club Escolar de Investigación y Producción Audiovisual y tenemos una unidad de dirección y una académica...", como también el docente Juan expresa "...Con los estudiantes iniciamos hacer pequeños proyectos de investigación: investigación en el aula, para cambiar los procesos de tele-secundaria por la enseñanza mediada por proyectos de investigación...". Con relación a esto, Saavedra et al. (2015) determinan que los semilleros de investigación son una estrategia extracurricular para la formación investigativa de los estudiantes, son grupos que propician espacios agradables, seguros y solidarios, además, generan un enriquecimiento voluntario académico y personal. Por otra parte, la investigación en la práctica va más allá de una asignatura como lo expresa el docente Juan:

“...iniciamos investigación focalizada en insectos, a partir de esta podíamos articular el $90 \%$ de todas las áreas de conocimiento, entonces pues ese proceso permitía no solo que el estudiante se motivara mas si no que viera que cada área no iba solo como por su lado si no que se podía integrar en un proceso en la resolución de un problema..."

Y el docente Marcos describe que:

“...el trabajo ambiental que veníamos haciendo en Guicán y que iniciamos en Puerto Boyacá lo configure en un proyecto que lo titulé Tras las Huellas del Agua, Conservamos Nuestro Territorio tiene tres líneas: Investigación, Educación Ambiental y Comunicación. La comunicación es a través de programa de radio, la producción de cortos documentales; La investigación, se sistematizó en un Libro que se titula Cuidando Al Planeta desde la Escuela; Educación Ambiental esa la tenemos a través de un decálogo que lo hemos llamado hogares 10 con el planeta. Tras las Huellas del Agua, trabajo desde cátedra de la paz, porque cátedra de la paz incluye el componente ambiental, la trabajo desde ética también y Ciencias Naturales...". 
En este sentido, la investigación realizada por los docentes es desarrollada de manera interdisciplinar, tomando de referencia a Flinterman, Teclemariam-Mesbah y Broerse (2001) la definen como una colaboración de varias disciplinas, es decir, de conceptos, metodologías o epistemologías que permiten un enriquecimiento mutuo al ser intercambiadas e integradas (citado en Henao et al., 2017).

Retomando lo expresado por el docente Juan: “...Cuando llegué a la zona a trabajar empezamos a hacer algunas salidas, me gustó mucho, porque es una zona con bastante bosque nativo, con aves, cuando uno salía, como los niños conocían la zona, empezaron espontáneamente a decir, cuidado profe con esto de ahí, cuidado profe que allí hay tal cosa, mire profe lo que hay; entonces, el proceso de investigación fue más empezar que ellos me enseñaran a mí, no yo a ellos. Se empezó a perfeccionar el lenguaje propio de las ciencias, permitiendo que ellos empezaran sus procesos de investigación, al involucrar a los papás, era chévere, porque íbamos a la casa de ellos, y nos poníamos a charlar, de plantas medicinales, logrando hacer un libro pequeño de plantas medicinales...", se evidencia que la práctica pedagógica del docente está acompañada de procesos investigativos y a la vez enriquecida por los conocimientos previos de los estudiantes y la experiencia de sus padres producto de la cotidianidad, dando lugar a la articulación con los conocimientos científicos abordados en la escuela, se concuerda con Melo (2017) ya que relaciona los conocimientos previos con las experiencias vividas que incluyen las actividades diarias de los padres y el reconocimiento del contexto. De esta manera, se evidencia un diálogo de saberes el cual requiere del educador una actitud reflexiva permanente, que le permita comprender su papel dentro de la comunidad educativa y social (Bastidas et al., 2009).

Por otra parte, el involucrar temas de interés de los estudiantes en los procesos investigativos, contribuye a estimular la automotivación posibilitando el despliegue de la creatividad, la inventiva y la generatividad de ideas y acciones (Tobón, 2006), como se expone en el siguiente fragmento: “...En Guicán de la Sierra, en la Normal Superior de Nuestra Señora del Rosario allá nos llamamos los Héroes del Planeta, empiezo aplicar la teoría de las inteligencias múltiples con los niños, entonces comienzo a trabajar de acuerdo con sus intereses, tengo los grupos: Shakira, Charles Darwin y Albert Einstein, dependiendo de su inteligencia las actividades se las dirigía hacia eso..." (docente Marcos).

La práctica pedagógica de los docentes define su autonomía cuando el perfil se alimenta de experiencias laborales, de inquietudes de formación autónomas y de una actualización constante de métodos y enfoques, facilitando y reconstruyendo el ejercicio de enseñar (Alarcón et al., 2019), esto se refleja en el relato de la trayectoria investigativa del docente Marcos:

“...El libro cuenta la historia de toda la estructura del club, tiene una unidad de dirección, una académica, donde los estudiantes se organizan en tres: semilleros, equipo de producción (los que tienen que ver con cámara, edición y toda esa parte) y los investigadores, quienes son los estudiantes líderes de los proyectos que hacemos; por ejemplo, nosotros tenemos los siguientes proyectos: (1). El Píleo, es el proyecto de lectura, escritura y oralidad y con ese proyecto trabajamos lectura en voz alta vinculando las familias, ese es el requisito para ingresar al Parche, en el Parche no tienen notas los estudiantes y es voluntario, al igual que cuando uno está en el grupo de investigación Gecos de la Universidad Pedagogica y Tecnológica de Colombia es voluntario; pero si tienen ese compromiso, de que van a leer en familia un cuento o novela por mes, y nosotros interactuamos con la biblioteca Nacional, y con la biblioteca pública municipal, pero en este ejercicio, la Biblioteca Nacional desde que yo estaba en Siachoque nos viene haciendo seguimiento y desde el año 2019 la Biblioteca Nacional de Colombia de Cultura, esta desarrollando algo que se Ilama el Programa Nacional de Bibliotecas Rurales Itinerantes, y la experiencia que tienen de referencia es la nuestra. (2). El otro proyecto es Fescol, entonces participamos, dependiendo de la temática. (3). Nosotros vamos con la consigna de que Nuevo Colón, va a hacer Patrimonio Paisajístico de Boyacá, por la parte frutícola de los caducifolios, y este proyecto tiene: aula 10, es el sendero de la institución educativa tiene 
una quebrada y desde ahí hacemos las 10 estaciones del decálogo hogares 10 con el planeta, entonces en la huerta, vamos a tener lo de las especies representativas de frutales de Nuevo Colón como un museo también frutícola; y arrancamos con lo del agua, la quebradita la Zorrera, y entonces me encuentro con que el colegio ya tiene planta de tratamiento de aguas residuales y tiene planta de tratamiento de agua potable, primer colegio de Boyacá que tiene las dos plantas, y la de agua potable que llega es la de esta quebrada (la zorrera) y aquí viene todo lo de la identidad. Es decir, la institución se convierte en ese paso de interpretación ambiental y con la concepción de que el aula no es un salón, el aula es la vereda, el aula es afuera; se acuerda de la revolución de los tres cambios, vincular a la escuela con su entorno, aula 10. (4). El otro proyecto que aquí trabajamos, es senderismo, entonces un domingo por mes, con los del Parche que nos quieran acompañar hacemos senderismos. Senderismo y ciclo montañismo van con lo de limpieza, recogiendo también basura. La otra actividad, que nosotros hacemos es una carrera de $6 \mathrm{~km}$ y $10 \mathrm{~km}$, la intención no es hacerla una sola vez al año, sino hacerla de pronto más veces y también en otras veredas. Esto también va con los hogares 10 en el planeta, el aula y los hogares, vinculando todo cuando hacemos ciclo montañismo, vamos haciendo el seguimiento casa a casa, de cómo, si su hogar es 6,5 debemos subirlo, cómo está el jardín, cómo está todo lo del agua; revisamos recibo del agua, recibo de luz, mirando consumo. (5). Y esto tiene el equipo de producción, el programa de radio que se llama Recreo al Aire, que salimos por Manzanal Stereo, en Siachoque lo hicimos a nivel del colegio porque en la emisora nos cobraban, en Nuevo Colón ya la alcaldía nos paga para que la emisora nos deje sacar el programa los miércoles a las 5 de la tarde media hora; y la producción de videos, el equipo de producción se encarga de hacer el registro de Fescol, el registro de Píleo y el registro de Nuevo Colón, Patrimonio Paisajístico de Boyacá, y finalmente esto si termina siendo escuela, y aplicando los tres cambios que hay que hacer en la escuela, haciendo que los estudiantes sean líderes, que sean ellos los que se empoderen, gestionen, que sean corresponsables, saliéndose de la parte de los contenidos, de la cuadrícula del horario de clase y vinculando la escuela con su entorno...".

En razón a lo anterior, se denota que la praxis del docente enmarca la importancia de la interdisciplinariedad del plan de estudios de la institución, como también la lectura de contexto que hace a partir de su proceso enseñanza aprendizaje, permitiendo a quienes aprenden desde sus realidades involucrar sus conocimientos previos y empíricos con conocimientos científicos develados en el aula de clase, de tal manera, que se extrapolan los saberes para generar una capacidad critica, reflexiva y propositiva de sus acciones conjuntas en pro del bien común, lo que implica que el docente debe convertirse en un ser crítico y reflexivo capaz de detectar sus propios problemas educativos y solucionarlos utilizando la investigación (Tomé, 2015).

\subsection{La Experiencia refleja los procesos investigativos}

Dentro de la experiencia docente es importante resaltar aquella motivada por la autosuperación, la cual los lleva a realizar cursos, indagar por lecturas y alternativas que mejoran su labor, y participar en seminarios, congresos y encuentros educativos (López, 2010). Dicha autosuperación se ve reflejada en la institución educativa, a partir de los procesos investigativos realizados, donde estudiantes y docentes participan en eventos y obtienen reconocimientos o premios que fortalecen la reflexión y dan cuenta de la experiencia; de acuerdo a esto, se resalta lo mencionado por el docente Marcos: “...empiezo por el lado del diario crónica de la semana en el año 2014, termino siendo experiencia en uso de las nuevas tecnologías en educar con Tic y eso me permitió estar en Bogotá y ya estando en Bogotá pase entre los 10 finalistas, estuvimos de ganadores y me fui para Corea en el año 2015 como experiencia Nacional en Innovación y Educar con Tic...", "...tras las Huellas del Agua, Conservamos Nuestro Territorio fue experiencia nacional en pragmática de la Educación Ambiental en el año 2018...", por otro lado, el docente Juan resalta: “...se presentaron algunos proyectos en Minciencias para participar con los estudiantes representando a Boyacá en la Feria Bio América Diversa en la ciudad de Manizales, en este evento participaron estudiantes de más de 11 países...", "...en la convocatoria Aventura Bio también fuimos 
seleccionados y estuvimos representando al departamento la reserva Otún Quimbaya, esta convocatoria premió a las 12 mejores instituciones oficiales del país que hayan tenido procesos largos de investigación en el aula...".

En este sentido, los procesos de investigación promovidos desde la escuela, trasciende en la vida de los estudiantes, en ocasiones determinando e influyendo en sus proyectos de vida, por ejemplo, hay estudiantes que escogen carreras relacionadas con los temas de investigación abordados en su época escolar, con el fin de replicar y mejorar lo aprendido para compartir y construir conocimiento:

“...algunos estudiantes tomaron la iniciativa, y uno de los líderes es el presidente de la junta de acción comunal, pero ellos conformaron el Nodo a nivel de Puerto Boyacá de la Red Nacional de Jóvenes de Ambiente, el estudiante se hizo responsable del grupo el Parche Caigua, y entre ellos también conformaron el Nodo de la Red Nacional de Jóvenes de Ambiente en el Guicán, y en Siachoque ocurrió lo mismo; los jóvenes estando en esto acogen la experiencia que veníamos haciendo y se convierten en líderes, en Siachoque la que fue líder, terminó siendo líder también de la Red de Jóvenes de Ambiente en Boyacá, y el de Guicán terminó siendo uno de los líderes de comunicaciones de la Red Nacional a nivel Nacional de Jóvenes de Ambiente; así se generan liderazgos con base en los procesos de investigación; a la vez, otros profesionales en diferentes áreas que llevan su sello verde, como su consigna, una hoy es Comunicadora Social en la universidad Santo Tomás y el otro es Abogado Especialista en Derecho Ambiental, ellos comenzaron estando en grado 5 y grado 6 respectivamente en la Normal..." (Marcos).

De acuerdo a esto, se evidencia que la curiosidad e inquietud por aprender nuevos enfoques y prácticas los lleva a no ser repetidores sino creadores de nuevas alternativas (López, 2010), no solo en estudiantes sino también en docentes, además, el apoyo y orientación de personas en el proceso de formación influye de manera considerable en sus aprendizajes y motivación, así lo menciona el docente Marcos:

"...el hecho de que a nivel de la universidad como lo que uno ve, es que, no es tanto lo que usted aprenda como tal en el aula, sino que es este tipo de experiencias, las pasantías, por ejemplo, la pasantía en Paz de Río, que genera otro ambiente, el compartir con otras entidades, y todo eso lo lleva a uno a no esperar a que me llegue, uno puede ir, hacer la gestión, llevar la carta, esperar 5 horas. Yo tengo 3 mentores, mi abuela, que fue docente en una época, que ella lo fue por saber leer y escribir; ya uno se va encontrando por el camino, digamos con la profesora de la universidad, que es mi mentora en este maravilloso mundo de la Investigación, y ya en la práctica, me encuentro con un docente de Puerto Boyacá...".

De igual forma, el docente Juan describe: “...tuve una docente mentor en mi formación en investigación, era la directora del Grupo Gecos, nos hacía ver ese proceso tan fácil, tan bonito, tan chévere, y que uno podía, no solo decir yo voy a viajar, sino que uno poder dirigirse ya a un público. Porque en el grupo, siempre más que un grupo hemos sido una familia, eso ha sido algo que la profe nos inculcó, aquí somos una familia, realmente le agradezco al grupo porque esto es lo que les enseño a mis estudiantes...".

Con lo anterior, se puede evidenciar que las diversas situaciones por la que pasa un docente, los lleva a abordar su labor educativa de una manera crítica, donde las vivencias con padres, familiares o amigos disciplinados y comprometidos socialmente se convierten en modelos de referencia para el aprendizaje de principios y valores, por consiguiente, se construyen profesionalmente con los años de experiencia docente sobre la base de sus experiencias (López, 2010).

\section{Conclusiones}

Se reconstruyó la trayectoria investigativa de dos docentes que se desempeñan en el área de Ciencias Naturales, en esta se articularon sus experiencias de acuerdo con la formación inicial y permanente, con la práctica pedagógica y el contexto desde el cual actúan. A su vez, se presentaron las bitácoras con los acontecimientos 
más representativos en la vida profesional de los docentes Juan y Marcos, donde se describe la prevalencia de procesos investigativos realizados a lo largo de su profesión.

Se evidenció que la formación investigativa, tanto inicial como permanente de los docentes participantes, ha jugado y viene jugando un papel importante en su práctica al brindarles las herramientas necesarias para orientar la enseñanza de las ciencias naturales de una manera interdisciplinar, participativa y contextualizada, al permitirles reflexionar sobre su acción para crear y recrear conocimiento e ir perfeccionando su rol.

La práctica pedagógica como escenario de investigación posibilita a los involucrados un aprendizaje continuo y cooperativo, en donde los participantes se ven beneficiados mutuamente; de esta manera, la formación investigativa del educador permite aprovechar el contexto para implementar diversas metodologías de enseñanza que faciliten el aprendizaje de los conocimientos disciplinares basados en los saberes previos y empíricos de los estudiantes, fortaleciendo y desarrollando habilidades, destrezas, hábitos, actitudes y valores que orientan los proyectos de vida de los mismos.

La investigación biográfica-narrativa como metodología, posibilitó la reconstrucción de la trayectoria investigativa de los docentes participantes, facilitando la descripción de experiencias, sentimientos, saberes, competencias profesionales y vivencias que son evocadas a través de un relato, al permitir reconocer la investigación como un elemento esencial en su quehacer docente. De igual manera, este estudio contribuye a una interrelación de que el sujeto se convierta en objeto de saber, rompiendo el paradigma de investigaciones educativas tradicionales con metodologías ya establecidas e incursionando en el juego de contar la verdad de sí.

\section{Referencias bibliográficas}

Aarón, G. M. A. (2016). El contexto, elemento de análisis para enseñar. Zona Próxima, (25),34-48. Recuperado de: https://www.redalyc.org/articulo.oa?id=853/85350504004

Alarcón, A., Montes, A. J., y Múnera, L. (2019). Fomento Institucional a la Investigación, Formación Docente y Prácticas Pedagógicas: un vínculo necesario en el programa de Derecho de la Universidad de CartagenaColombia. Revista Espacios, 40(8), 26. Recuperado de: https://revistaespacios.com/a19v40n08/19400826.html

Araque-Suárez, C. (2019). Los restos del mundo globalizado, al docente investigador universitario y su práctica pedagógica. Aibi: Revista de Investigación, Administración e Ingeniería, 7(2), 50-56.

Arias-Cardona, A. M., y Alvarado-Salgado, S. V. (2015). Investigación narrativa: apuesta metodológica para la construcción social de conocimientos científicos. CES Psicología, 8(29, 171-181.

Bastidas, M., Pérez, F., Torres, J., Escobar, G., Arango, A., y Peñaranda, F. (2009). El diálogo de saberes como posición humana frente al otro: referente ontológico y pedagógico en la educación para la salud. Invest Educ Enferm, 27(1), 104-111.

Bolaños, J. (2016). El docente ante el oficio de investigador en el aula. Praxis y Saber, 7(15), 63-80. DOI: http://dx.doi.org/10.19053/22160159.v7.n15.2016.5723

Bolívar, A. Domingo, J. Fernández, M. (2001). La investigación biográfico-narrativa en educación. Enfoque y metodología. Madrid: Editorial La Muralla, S. A.

Díaz-Quero, V. (2006). Formación docente, Práctica Pedagógica y Saber Pedagógico. Laurus, 12 (Ext.), 88-103. Recuperado de: https://www.redalyc.org/pdf/761/76109906.pdf 
Duarte, J. (2007). Formación permanente de docentes en servicio, Alternativa para la enseñanza y el aprendizaje de la Lengua escrita en la Educación Básica Integral. Unisersitat Rovira I Virgili. Recuperado de: https://dialnet.unirioja.es/servlet/tesis?codigo=77631

Fernándes, C. (2014). Relaciones entre la Investigación y la formación docente permanente: el conocimiento necesario para la diversidad. Estudios Pedagógicos, XL(2), 161-174.

Giraldo, G. E. (2013). Acerca de la lectura de contexto. En finalidad: Documento de apoyo para el Programa de Formación sobre Desarrollo y Articulación de Proyecto Pedagógicos Transversales- Ser con Derechos. Convenio Universidad de Antioquia - Ministerio de Educación Nacional. Recuperado de: https://aprende.colombiaaprende.edu.co/ckfinder/userfiles/files/Lectura\%20de\%20Contexto.pdf

González, N., Zerpa, M., Gutiérrez, D., y Pirela, C. (2007). La investigación Educativa en el Hacer Docente. Laurus, 13 (23), 279-309.

Henao, C., García, D., Aguirre, E., González, A., Bracho, R., Solorzano, J., y Arboleda, A. (2017). Multidisciplinariedad, interdisciplinariedad y transdisciplinariedad en la formación para la investigación en ingeniería. Revista Lasallista de Investigación, 14 (1), 179-197. Recuperado de: https://www.redalyc.org/pdf/1995/199553113014.pdf

López, S. (2010). Historia de vida de buenos profesores: experiencia e impacto en las aulas. Profesorado: Revista de Currículum y formación del profesorado, 14 (3), 149-164. Recuperado de: http: //www.ugr.es/local/recfpro/rev143ART10.pdf

Manzano-García, B. (2015). La Investigación en Educación llevada a la práctica para la mejora en los procesos educativos. Tomé-Fernández, M., y Manzano-García, B (es), Investigación en la práctica docente, 5-16. Zaragoza. España: Terue.

Mélich, J. C., y Bárcena, F. (2014). La educación como acontecimiento ético. Natalidad, narración y hospitalidad. Barcelona: Paidós. p. 99-134 (Cap. 3. Paul Ricoeur: Educación y narración).

Melo-Brito, N. (2017). Los puentes en la enseñanza de las ciencias: un compromiso para comprender las investigaciones sobre las relaciones entre conocimientos científicos escolares y conocimientos ecológicos tradicionales. Tecné Episteme y Didaxis: TED, $43-61$. Recuperado de: http://www.scielo.org.co/pdf/ted/n42/0121-3814-ted-42-00043.pdf

MEN. (2013). Sistema Colombiano de Formación de Educadores y Lineamientos De Política. Ministerio de Educación Nacional. Bogotá Colombia. Recuperado de: https://www.mineducacion.gov.co/1759/articles345485_anexo1.pdf

Minciencias. (2020). Cultura en CTel. El Conocimiento es de todo. Ministerio de Educación Nacional. Colombia. Consultado en: https://minciencias.gov.co/cultura-en-ctei/ondas

Minciencias. (2020). Grupos de Investigación Reconocidos. La Ciencia en Cifras. EL conocimiento es de todos. Ministerio de Educación Nacional. Colombia. Consultado en:https://minciencias.gov.co/la-ciencia-encifras/grupos

Muñoz-Martínez, M., y Garay, F. (2015). La Investigación como forma de desarrollo profesional docente: Retos y Perspectivas. Estudios Pedagógicos, XLI(2), 389-399.

Paéz, R. (2015). Experiencia, Práctica y Saber Pedagógicos. Campo analógicos y Posibilidades para el Maestro. En R. Paéz (Ed), Práctica y Experiencia: claves del saber pedagógico docente. Bogotá, Colombia: Ediciones 
UniSalle. Recuperado de: http://biblioteca.clacso.edu.ar/Colombia/fceunisalle/20170117095042/Practicaexp.pdf

Parga, D. (2016). El Continuo de la formación del profesorado en Ciencias. TED, 40, 1-15.

Peláez-Ruíz, A. M., y Piedrahita-Marín, D. A. (2018). La importancia del docente investigador en las aulas de primaria, básica y media: una reflexión a partir de dos estudios. Revista Reflexiones y Saberes, (9), 40-48.

Plata, M. (2015). Formación en Investigación en el Departamento de Boyacá: Aportes del Programa Ondas Minciencias. Praxis y Saber, 7(15), 103-125. DOI: http://dx.doi.org/10.19053/22160159.v7.n15.2016.5725

Porlán, R. (2011). El maestro como investigador en el aula. Investigador para conocer, conocer para enseñar. Universidad Nacional Atónoma de México. p. 25-45. UNAM-México.

Ricoeur, P. (1996) Si mismo como otro. Madrid. Siglo XXI.

Saavedra-Cantor, C. J., Muñoz-Sánchez, A. I., Antolínez-Figueroa, C., Rubiano-Mesa, y L., Puerto-Guerrero, A. H. (2015). Semilleros de investigación: desarrollos y desafíos para la formación en pregrado. Educación y Educadores, 18 (3), 391-407. DOI: http://dx.doi.org/10.5294/edu.2015.18.3.2

Tobón, S. (2006). Método de trabajo por proyectos. Madrid: Uninet

Tomé-Fernández, M. (2015). El docente como investigador. Tomé-Fernández, M., y Manzano-García, B (es), Investigación en la práctica docente, 19-26. Zaragoza. España: Terue.

Vázquez, Á., y Monassero, M. (2019). La educación de Ciencias en Contexto: aportaciones a la formación del profesorado. TED, 46, 15-37.

Yedaide, M. M., Álvarez, Z., y Porta, L. (2015). La investigación narrativa como moción epistémico-política. Revista Científica Guillermo de Ockham, 13(1), 27-35

Esta obra está bajo una Licencia Creative Commons Attribución-NoCommercial 4.0 International

$(\mathrm{Cc}) \mathrm{BY}$-NO 